Relations industrielles

Industrial Relations

\title{
Bernatchez, Jean-Claude, La résolution des griefs dans \\ l'entreprise
}

\section{Hélène Gascon}

Volume 50, numéro 3, 1995

URI : https://id.erudit.org/iderudit/051044ar

DOI : https://doi.org/10.7202/051044ar

Aller au sommaire du numéro

Éditeur(s)

Département des relations industrielles de l'Université Laval

ISSN

0034-379X (imprimé)

1703-8138 (numérique)

Découvrir la revue

Citer ce compte rendu

Gascon, H. (1995). Compte rendu de [Bernatchez, Jean-Claude, La résolution des griefs dans l'entreprise]. Relations industrielles / Industrial Relations, 50(3),

668-670. https://doi.org/10.7202/051044ar

Tous droits réservés @ Département des relations industrielles de l'Université Laval, 1995
Ce document est protégé par la loi sur le droit d'auteur. L’utilisation des services d'Érudit (y compris la reproduction) est assujettie à sa politique d'utilisation que vous pouvez consulter en ligne.

https://apropos.erudit.org/fr/usagers/politique-dutilisation/ 
In Part 4 the authors provide a comprehensive summary of their conclusions. In this section the authors offer explanations for the generational impact on values. Kelly and Heery argue that many of their findings can be traced to the fact that a number of contemporary officers were shop stewards during the 1960 s and 1970s. This was a period when stewards exhibited a great deal of militancy. Thus strong support for workplace organization, a belief in the need for steward independence, encouragement of ambitious bargaining objectives and a deep mistrust of management all flow from the strong workplace organization and adversarial industrial relations system present in Britain in the 1970s. The authors state that if their conclusions about generational impact on values hold true then it is fair to ask whether officers appointed in the near future will show less militancy than current officers. Kelly and Heery argue that it may well be that - especially in the private sector where strike incidence has declined greatly during the 1980 s newer officers will display a greater willingness to cooperate with management than did their predecessors.
In conclusion this book belongs on the shelf of every serious industrial relations scholar. It should be required reading for graduate students. The main theme of this volume, that values-as determined by age or generational differences-determine the activities of trade union officers has tremendous implications for trade unionists and trade union scholars in North America. North American scholars studying trade unions now have a blue print to follow in designing a study to analyze factors determining the actions of North American trade union officers. Much of the North American research in the area of trade union governance starts out with acceptance of bureaucracy theory as an explanation for how trade unions function. From my perspective the most important contribution of this volume is to force North American scholars to reconsider this assumption. Kelly and Heery have laid out the path, it is now up to us to follow it and begin an in-depth parallel study of trade union officers on this continent.

NORMAN A. SOLOMON University of Windsor

\section{La résolution des griefs dans l'entreprise}

par Jean-Claude BERNATCHEZ, Trois-Rivières, CIRIM Éditeur, 1994, 428 p., ISBN 2-9802036-5-3.

Cet ouvrage vise à décrire les mécanismes de résolution des litiges (MRL) en contexte syndiqué ou non syndiqué. Le premier chapitre expose un cadre de référence dans lequel les litiges sont définis comme étant des plaintes ou des griefs qui surviennent lorsqu'il y a un écart sur la façon de percevoir une situation et de la résoudre. Des personnes sont mandatées pour régler ces litiges et trouver une solution durable en autant que les réalités internes de l'entreprise aient été prises en considération, notamment, les valeurs organisationnelles, les droits et les obligations des parties.
Le chapitre 2 porte sur les griefs et la gestion des ressources humaines (GRH). Les domaines étudiés dans cette partie touchent la période de probation des nouveaux employés, la dotation, l'ancienneté, les mouvements de personnel, la rémunération globale et la santé au travail. Les gestionnaires sont préoccupés par ces domaines de la GRH, puisqu'ils ont des conséquences sur la productivité des personnes et de l'organisation, en plus de faire fréquemment l'objet de griefs selon l'auteur.

Le chapitre 3 met en lumière la conduite des parties, en vue de maintenir des relations de travail efficaces sur 
les lieux du travail. On y traite donc d'équité ou du respect des droits des employés, de l'exercice de l'autorité patronale, de l'obéissance des salariés, ainsi que de l'imposition de mesures disciplinaires ou non disciplinaires selon les actes reprochés. Les principaux sujets qui préoccupent les entreprises sont aussi abordés très brièvement comme l'absentéisme, le vol ou la fraude, l'incapacité du salarié, le harcèlement sexuel ou la discrimination. Enfin, sont expliquées les pratiques en vigueur dans un lieu de travail, c'est-à-dire les coutumes, les privilèges et les droits acquis.

Le chapitre 4 porte sur la gestion des griefs à l'interne issus de la convention collective. L'accent est mis sur l'importance d'étudier les griefs selon une approche de solution de problèmes pour éviter le recours systématique à l'arbitrage. Le modèle traditionnel de négociation et de résolution des litiges fondé sur des tactiques d'information, de persuasion et de coercition, ainsi que sur des stratégies de bluff et de sincérité, est donc remis en cause au profit d'une approche fondée sur un échange rationnel entre les parties considérées comme des partenaires et non pas comme des adversaires.

Les chapitres 5 à 7 décrivent le mécanisme de l'arbitrage des griefs, le processus de l'audition et la fonction de l'arbitre quant à la prise de décision. L'arbitrage des griefs est ici qualifié de mode traditionnel de résolution des litiges liés à la convention collective. $\mathrm{Ce}$ mode se différencie de l'arbitrage dit non conventionnel de plus en plus utilisé, depuis 1980, par la promulgation de lois d'ordre public (ex.: Loi sur les normes du travail, L.R.Q., c. N-1.1).

Dans les deux derniers chapitres sont présentés différents types de MRL. Les recours internes comprennent la politique de porte ouverte et les tribunaux internes à l'organisation composés de représentants des salariés et de l'employeur. L'ombudsman, la médiation pré- ventive, la médiation pré-arbitrale et l'arbitrage non syndical sont des recours externes. Les grandes organisations non syndiquées sont les plus susceptibles de recourir à ces types de MRL.

Dans l'ensemble, ce livre permet d'avoir un bon aperçu du fonctionnement et de l'utilité des MRL mis sur pied par les organisations dont le personnel est syndiqué ou non syndiqué. Quelques commentaires spécifiques sont rapportés ci-dessous quant à certaines parties de ce livre.

D'abord, il est précisé dans l'introduction que les litiges sont assimilés à des plaintes et à des griefs et que le différend relié à la négociation collective a été exclu de l'objet principal de l'étude. Cet ouvrage aurait donc pu s'intituler "La résolution des litiges dans les organisations " ou "La résolution des griefs et des plaintes dans les organisations " pour bien refléter son contenu.

D'autre part, il est mentionné au chapitre 2 que "les griefs sont issus fréquemment de problèmes liés à la gestion des ressources humaines (p. 64). Toutefois, ces problèmes reliés à la gestion des personnes et des emplois sont discutés avant même d'avoir abordé le vif du sujet, soit la notion de griefs relatifs à l'interprétation et à l'application de la convention collective. À mon point de vue, ce chapitre coupe le fil conducteur de cet ouvrage. Comprendre la notion de griefs et le mécanisme de l'arbitrage des griefs aurait été très souhaitable avant de traiter de sujets particuliers qui peuvent faire l'objet de griefs.

Le résumé du troisième chapitre reprend au paragraphe 2 (p. 175) des notions qui ont été discutées déjà au chapitre 1, soit l'approche des droits résiduaires et l'approche des droits institutionnels. Le résumé du chapitre 1 (p. 60) et non pas du chapitre 3 devrait $y$ faire référence.

Les chapitres 4 à 7 se succèdent selon une bonne séquence en ce qui 
concerne l'étude des griefs et de l'ensemble du mécanisme de l'arbitrage des griefs. Évidemment, la publication du livre a eu lieu avant que de nouvelles règles législatives ne s'appliquent, notamment, en ce qui a trait à la durée de la convention collective. (À ce sujet, voir Rodrigue Blouin et Fernand Morin, Droit de l'arbitrage de grief, $4^{\mathrm{e}}$ édition, Cowansville, Éditions Yvon Blais, 1994, p. 52.) D'autre part, sous les sous-titres "Convention exclusive " (p. 186) ou " Convention effective et stable" (p. 187), il aurait été pertinent de citer les articles du Code du travail (L.R.Q., c. C-27) faisant référence à ces notions d'exclusivité et de durée de la convention collective. Le même commentaire peut s'appliquer à d'autres endroits (exemple : la sentence est sans appel et lie les parties, p. 332).

Quant aux deux derniers chapitres, l'exposé sur les MRL et leur raison d'être est un point de départ. Certaines données déjà existantes ou à collecter pourraient davantage nous renseigner sur les organisations qui ont mis sur pied des MRL au Québec, au Canada ou dans d'autres pays, actuellement. Quelques exemples sont mentionnés comme Northrup (1946), General Electric, Trans World Airlines et Control Data à partir de publications datant de 1984 (p. 354 368 ), ce qui pourrait être mis à jour ultérieurement.

Par ailleurs, je n'ai pas vérifié toutes les sources de jurisprudence mentionnées par l'auteur dans son analyse. Je présume que celles-ci furent choisies avec soins. Je signale enfin quelques petites corrections de forme. La plus importante concerne les références législatives comme les Chartes des droits individuels, la Charte de la langue française ou le Code civil. À cet effet, des règles sont prévues pour citer les lois et elles n'ont pas été appliquées. En outre, l'usage de certaines expressions sont à revoir comme " monter dans l'entreprise " (p. 63), "lancer un MRL" (p. 389) ou "politiques organisationnelles versus de relations avec les employés" (p. 50). Aussi, la référence de Trisler, 1984 (p. 368) n'est pas mentionnée dans la bibliographie.

Cet ouvrage permet donc de s'initier aux aspects techniques et théoriques de l'étude des mécanismes de résolution des griefs et des plaintes dans les organisations. L'étude de l'arbitrage des griefs occupe une part importante du volume.

HÉLÈNE GASCON

Université du Québec à Rimouski

\section{La qualité totale: nouvelle panacée du secteur public? \\ par Gérard ÉTHIER, Sainte-Foy, Qc, Presses de l'Université du Québec, 1994, 220 p., ISBN 2-7605-0758-0.}

Essentiellement, l'ouvrage décrit et analyse des expériences d'implantation de projets qualité dans cinq organisations publiques, bien ciblées et complémentaires: l'Hôpital du Haut-Richelieu, l'Hôpital Maisonneuve-Rosemont, la ville de Charlesbourg, le ministère de l'Industrie, du Commerce et de la Technologie et la Commission scolaire Saint-Jérôme. La description de ces cas s'effectue en cinq chapitres précédés par une partie intitulée: "Cadre théorique et méthodologique ", le tout complété par un der- nier chapitre ayant trait à la synthèse finale.

Les cas pratiques sont décrits dans un premier temps et sont suivis d'analyses et de commentaires. L'évaluation est surtout d'ordre psychosocial concernant différents paramètres inhérents à la gestion du changement : résistance, approche-client, sentiment d'appartenance, participation, responsabilisation, communication, motivation du personnel, visibilité des gestes, leadership, etc. Cette contribution empirique présentée avec un bon 